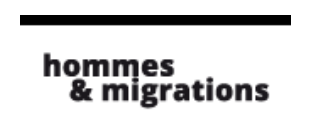

\section{Hommes \& migrations}

Revue française de référence sur les dynamiques

migratoires

1319 | 2017

Réfugiés et migrants au Liban

\title{
Mutation du rôle de la femme rurale réfugiée en zone urbaine
}

Le cas des femmes chrétiennes irakiennes au Liban

\section{Raphaël Paul Koupaly}

\section{OpenEdition}

Journals

Édition électronique

URL : http://journals.openedition.org/hommesmigrations/3968

DOI : ERREUR PDO dans /localdata/www-bin/Core/Core/Db/Db.class.php L.34 : SQLSTATE[HY000]

[2006] MySQL server has gone away

ISSN : 2262-3353

Éditeur

Musée national de l'histoire de l'immigration

Édition imprimée

Date de publication : 1 octobre 2017

Pagination : 57-64

ISBN : 978-2-919040-39-1

ISSN : $1142-852 X$

\section{Référence électronique}

Raphaël Paul Koupaly, " Mutation du rôle de la femme rurale réfugiée en zone urbaine », Hommes \& migrations [En ligne], 1319 | 2017, mis en ligne le 01 octobre 2020, consulté le 08 janvier 2021. URL http://journals.openedition.org/hommesmigrations/3968; DOI : https://doi.org/10.4000/

hommesmigrations.3968 


\section{MUTATION DU RÔLE \\ DE LA FEMME RURALE RÉFUGIÉE EN ZONE URBAINE}

\section{LE CAS DES FEMMES CHRÉTIENNES IRAKIENNES AU LIBAN}

Par RAPHAËL PAUL KOUPALY, doctorant, anthropologue, chercheur au Centre d'études pour le monde arabe moderne (CEMAM), université Saint-Joseph de Beyrouth ${ }^{1}$.

Le nombre de familles chrétiennes originaires d'Irak et réfugiées au Liban a triplé depuis 2014 et la chute de la plaine de Ninive passée au main du groupe «État islamique ». Pour ces 6500 familles d'origine rurale, installées majoritairement dans les régions côtières du Liban, la migration forcée a entraîné une recomposition des cellules familiales et une redistribution des rôles genrés. La tendance est à l'effritement de la famille patriarcale traditionnelle au profit de familles nucléaires où les femmes jouent un rôle social déterminant : face aux hommes au chômage et en situation illégale, c'est sur elles que repose désormais la survie du foyer.

Depuis la création de l'État irakien en 1921, le Liban a reçu diverses vagues migratoires d'Irakiens fuyant les changements politiques, militaires et religieux qui secouent leur pays. Auparavant pays de refuge pour les différentes communautés originaires d'Irak, le Liban est devenu, surtout depuis les années 1970, leur principal pays de transit. Les années 1980 et 1990 ont connu une vague migratoire composée majoritairement de jeunes hommes fuyant la conscription obligatoire lors des guerres successives contre l'Iran (1980-1988), le Koweït (1990-1991), la coalition internationale (1991)2, puis lors de l'imposition de l'embargo international (1991-2003)3. Cependant, la migration contemporaine la plus importante qui a touché l'Irak commence en $2003^{4}$, suite à la chute de Saddam Hussein et du parti Baath. Elle est de nature familiale et concerne toutes les communautés

1. Cet article est le fruit d'une étude sociologique effectuée auprès des réfugiés chrétiens irakiens entre mars et juillet 2016 par l'Évêché chaldéen de Beyrouth en collaboration avec le département de sociologie de l'université Saint-Joseph de Beyrouth. 2. Coalition de plus de trente pays mandatés par l'ONU qui intervient militairement en Irak et au Koweït en 1990-1991 avec comme objectif la destruction du potentiel militaire de l'Irak et la libération du Koweït. 3. https://www.lorientlejour.com/article/1027613/le-liban-secoue-par-des-vaguessuccessives-de-refugies.html 4. "Ce n'est pas tant le conflit de 2003 en tant que tel qui a entraîné le départ de nombreux réfugiés, mais plutôt la dégradation sécuritaire qui s'est développée par la suite, ainsi que les difficultés économiques qui se sont aggravées. Le pic d'arrivé de réfugiés irakiens se situe entre 2006 et 2008. "Mohamed Kamel Doraï, "Le rôle de la Syrie dans l'accueil des réfugiés irakiens depuis 2003 : espace de transit, espace d'installation », in Méditerranée, n¹13, 2009 [en ligne]. 
religieuses irakiennes, mais surtout les minorités ${ }^{5}$ : chrétiens orientaux ${ }^{6}$, Yézidis ${ }^{7}$ et Sabéens ${ }^{8}$. En effet, selon Cyril Roussel, "ces groupes confessionnels, faibles démographiquement par rapport au triptyque chiite/sunnite/kurde, n'ont pas leurs propres milices en Irak et sont physiquement menacés ${ }^{9}$.

L'exode des minorités culmine en 2014 après la chute de la plaine de Ninive $e^{10}$, un des foyers ruraux principaux des groupes de minorités en Irak, aux mains du groupe «État islamique " (Daesh). Les 150000 Chrétiens recensés en $2012^{11}$ dans les villages de la plaine de Ninive fuient vers le Kurdistan Irakien et s'installent temporairement dans les camps de déplacés autour de Erbil et de Dohuk. Dans les différents flux migratoires depuis 1921, les chrétiens irakiens forment le groupe le moins enclin au retour, comme le montrent les statistiques des différentes églises en Irak $^{13}$ et de la diaspora irakienne dans les pays occidentaux au fil des années. Selon un article du journal français $L a$ Croix en 2012 : «En 1987, l'Irak comptait 1,2 million de chrétiens. $70 \%$ deschrétiens d'Irakont donc quittéleur pays depuisla guerre de 200314. » En 2017, le nombre des chrétiens vivant en Irak ne dépasse plus les 350000 .

Installés de façon temporaire au Liban, dans les banlieues populaires au nord et à l'est de Beyrouth, en tant que "réfugiés ${ }^{15}$ » inscrits au Haut Commissariat des Nations unies pour les réfugiés (HCR), ils sont des candidats potentiels au

\section{/// Les villages chrétiens de la plaine de Ninivel envahis par le groupe « État islamique » (Daesh)}

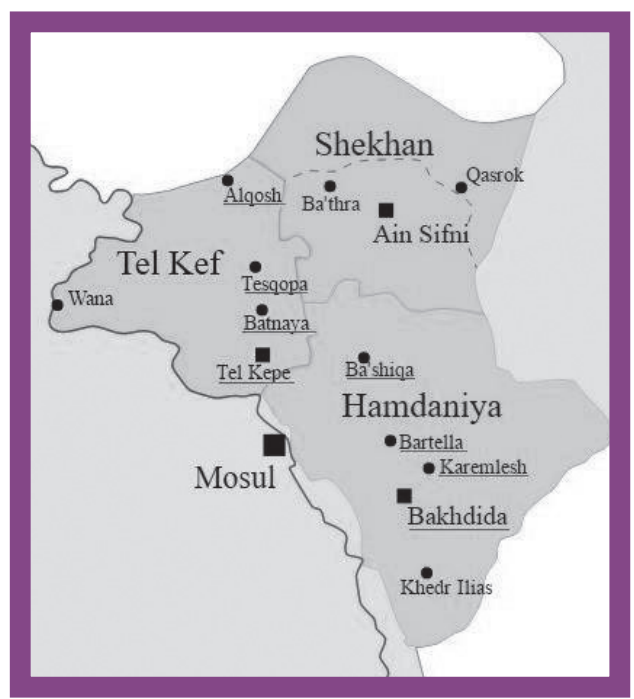

Les villages chrétiens conquis par le groupe "État islamique " (Daesh) en 2014 sont soulignés sur la carte : Tesqopa, Tel Kepe, Batnaya, Alqosh, Karemlesh (Chaldéens) et Ba'shiqa, Bartella, Bakhdida (Syriaques) et d'autres petites bourgades dans leurs alentours.

Source: Wikipedia. Url : https://fr.wikipedia.org/wiki/Plaine_de_ Ninive\#/media/File:Nineveh_Plains.svg.

programme de réinstallation (Resettlement Plan $\left.{ }^{16}\right)$. Ils vivent dans un milieu urbain à majorité chrétienne. Cependant, face aux changements

5. Selon l'UNHCR, l'Irak compte, en 2003, $88 \%$ d'Arabes et $12 \%$ de minorités ethniques, dont $5 \%$ de chrétiens. 6 . Chaldéens, Syriaques Orthodoxes et Catholiques, Assyriens, Arméniens Orthodoxes et Catholiques. 7. "Êzidî » en kurde, membres d'une minorité religieuse, du sud et de l'est de l'Anatolie, du nord de l'Iraq, de la Syrie et de l'Iran, avec des petits groupes en Arménie et Géorgie. Ils parlent un dialecte kurde et pratiquent une religion monothéiste qui mêle des éléments du christianisme, de l'islam et d'autres religions du Proche-Orient, et dont l'un des dogmes est la réhabilitation de l'ange déchu Malak Taous (L’Ange Paon). 8. Adeptes du mandéisme, religion baptiste, monothéiste et gnostique qui ne compte plus que quelques milliers de membres vivant dans les régions marécageuses autour des rivières en Irak et en Iran. Leur langue est un dialecte araméen. 9. Cyril Roussel, "Jeramana, voisine de Damas : le devenir d'une ville réceptacle de réfugiés irakiens", in Revue géographique de l'Est, vol. 49, n 4, 2009 [en ligne]. 10. Plaine qui se situe dans la province de Ninive en Irak au nord-est de Mossoul, également connue comme plaine de Mossoul (syriaque : "Dasta d'Ninwe "; arabe : sahl naynawa ". Formée de trois districts : Tel Kepe, Al-Hamdaniya, et Ain Sifni, elle compte des villages chrétiens, yézidis, kurdes et arabes. 11. Voir "Les chrétiens d'Irak et d'Orient en chiffres", in La Croix, 5 avril 2012 [en ligne]. 12. Les villages chrétiens conquis par le groupe "État islamique" (Daesh) en 2014 sont soulignés sur la carte : Tesqopa, Tel Kepe, Batnaya, Alqosh, Karemlesh (Chaldéens) et Ba'shiqa, Bartella, Bakhdida (Syriaques) et d'autres petites bourgades dans leurs alentours. 13. En 2016, on compte à peu près 350 ooo chrétiens restés en Irak (à Bagdad, Basra, Kirkūk et Kurdistan Irakien) contre environ 1 million dans la diaspora, principalement Aux États-Unis, en Australie, au Canada et en Europe de l'Ouest. 14. Voir "Les chrétiens d'Irak et d'Orient en chiffres ", op. cit.

15. Le Liban n'est pas signataire de la convention de 1951 relative au statut des réfugiés, ni du protocole de 1967 , laissant la loi de 1962 relative à l'entrée et au séjour des étrangers en tant que facteur déterminant du statut juridique des Irakiens installés au Liban. 16. Programme dirigé par l'UNHCR qui consiste à transférer des réfugiés d'un pays d'asile à un autre État qui a accepté de les admettre et de leur accorder à terme une résidence permanente. Seuls quelques États participent au programme de réinstallation. Depuis ces dernières années, les États-Unis d'Amérique étaient le principal pays de réinstallation dans le monde, tandis que le Canada, I'Australie et les pays nordiques offrent également un nombre annuel de places relativement important. Url : http://www.unhcr.org/fr/ reinstallation.html. 
sociaux, culturels et religieux radicaux, ils subissent une crise identitaire due à leur déracinement et aux difficultés d'intégration dans leur nouveau milieu. Un rapport de 2014 du Centre des migrants de Caritas Liban ${ }^{17}$ indique que $71,8 \%$ des réfugiés affirment se trouver dans une situation économique plus difficile que celle qu'ils avaient lorsqu'ils vivaient en Irak, et 66 \% estiment qu'ils sont à présent plus pauvres qu'au moment de leur arrivée au Liban. Ils se plaignent du taux élevé du chômage, des bas salaires, de la hausse des loyers et du coût des produits de première nécessité.

Les premiers îlots d'installation des réfugiés chrétiens irakiens (1991-2014) se trouvaient au quartier des Assyriens «Hay Al Ashouriyin ${ }^{18}$ » à Sad el Bouchrieh et ses environs. Hommes seuls ou familles nucléaires, ils étaient issus dans leur majorité des zones urbaines de Bagdad et de Mossoul.
Depuis 2014, le territoire de résidence des migrants chrétiens irakiens s'est élargi, au gré des nouvelles migrations, issues en majorité de familles patriarcales traditionnelles des villages de la Plaine de Ninive à l'est et au nord de la ville de Mossoul. Comme le souligne le $\mathrm{HCR}$, «les exilés s'installent généralement dans les quartiers les plus défavorisés des villes $^{19}$ ». C'est pourquoi, la majorité des réfugiés (80\%) habite les quartiers des municipalités de Jdeidet el Metn-Bouchrieh-Sad el Bouchrieh et Dekweneh (Sad el Bouchrieh, Sabtié, Rawda, Zaatriyé, Ras el Dekweneh, etc.) avec des petits groupes dans les villes avoisinantes de Sin el Fil et Bourj Hammoud, dans le Caza du Metn Nord, des banlieues populaires à majorité chrétienne au Nord-Est de la ville de Beyrouth.

D'autres petits groupes se sont délocalisés vers les autres villes de la zone urbaine à l'Est et au Nord de Beyrouth tels Hazmieh, Hadath, Baabda, Jounieh, Amchit et ailleurs au Liban.

\section{/// Nombre de familles de réfugiés chrétiens irakiens au Liban}

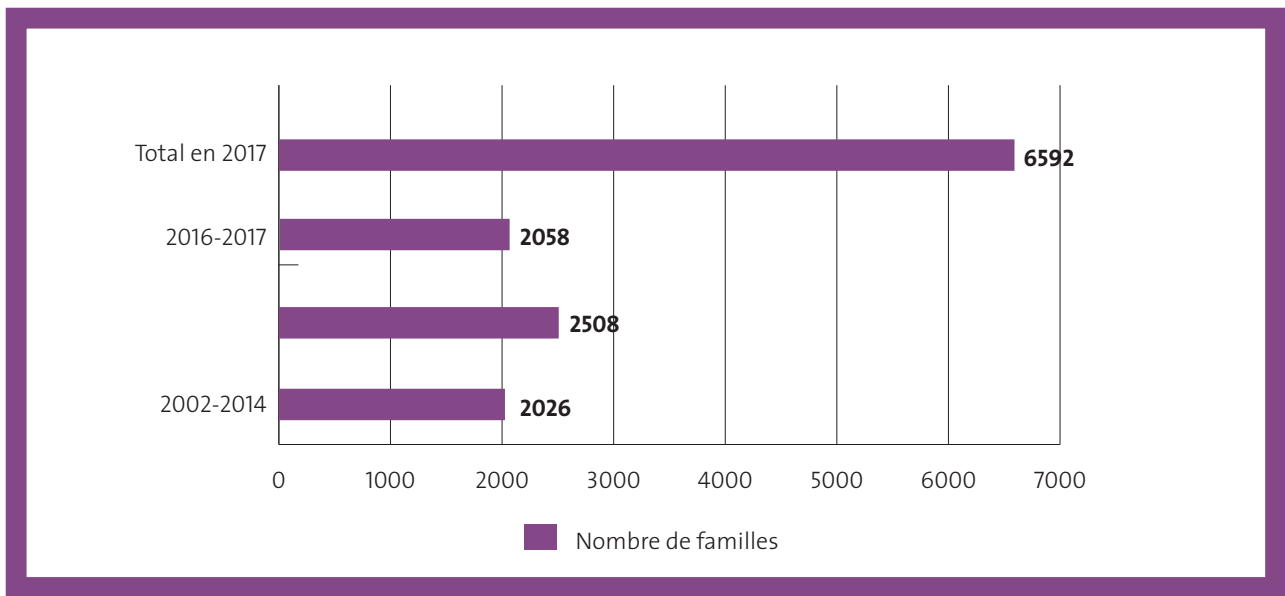

Source : Registres des églises chaldéenne, syriaque orthodoxe et catholique, assyrienne et arménienne orthodoxe. 


\section{/// Les principaux foyers de résidence des réfugiés chrétiens irakiens (Banlieue Nord-Est de Beyrouth)}
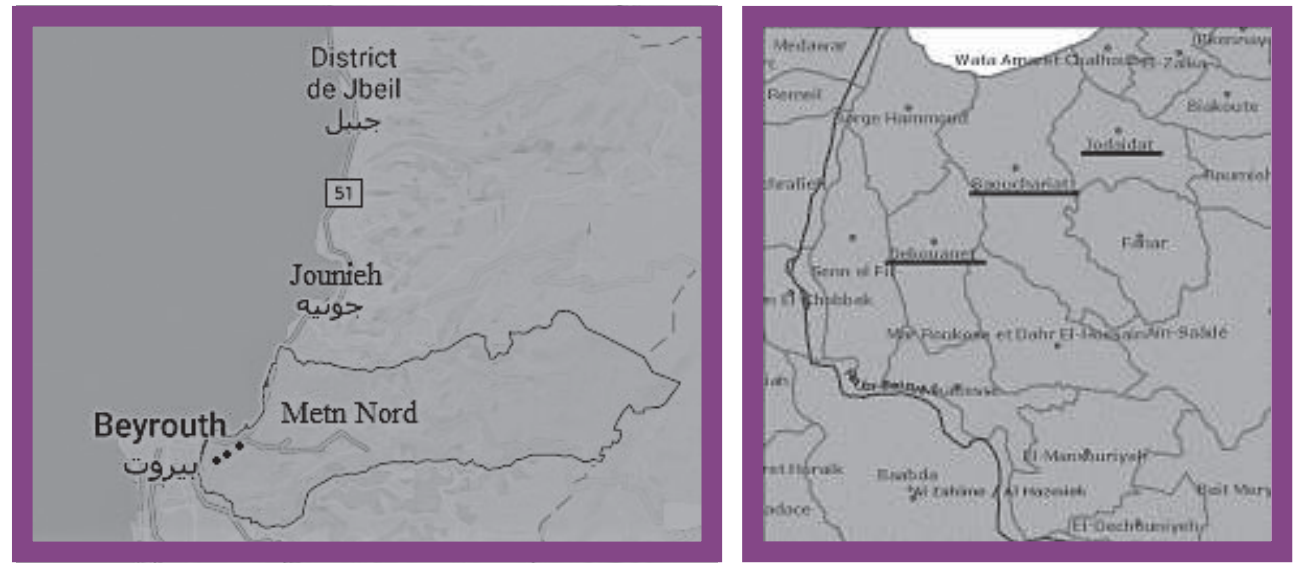

Source : http://www.localiban.org/article4516.html.

Vers la fin 2015, suite à l'installation en milieu urbain, une tendance à l'effritement de la famille patriarcale traditionnelle en familles nucléaires ou monoparentales est observée. De même, les femmes, auparavant reléguées à un rôle second dans les zones rurales du pays d'origine, acquièrent un rôle de plus en plus important dans la direction des ménages et dans le domaine du travail.

\section{La situation de la femme chez les chrétiens de la plaine de Ninive avant 2014}

En Irak, avant 2014, les familles chrétiennes dans les villages de la plaine de Ninive sont patriarcales ${ }^{20}$ et patrilocales ${ }^{21}$, à l'image de la société arabe tribale avoisinante, travaillant en majorité dans l'agriculture et l'élevage. Le village (appelé "Mata» qui signifie « Terre » en langue syriaque) se divisait en «Hay» (" quartiers »). Chaque quartier appartenait à un clan et chaque clan se divisait en différentes familles patriarcales. Le seul lieu commun à tous restait l'église, qui se trouvait dans la plupart des cas au centre du village.

Les populations des villages chrétiens de la plaine de Ninive étaient homogènes sur le plan religieux. Toutefois, il y avait quelques villages avec des minorités musulmanes ou yézidies qui habitaient en général dans des quartiers réservés aux abords du village pour éviter toute mixité indésirable. Au sein des familles patriarcales chrétiennes rurales, le rôle de la femme était limité au foyer familial, comme pour la majorité des femmes ${ }^{22}$ dans les sociétés rurales irakiennes.

La langue parlée localement dans la vie quotidienne dans les villages est le soureth, une langue vernaculaire issue du syriaque, avec des accents différents selon le village. L'arabe, langue officielle, restait limité à l'enseignement et aux contacts avec l'entourage musulman. Les classes de catéchisme et les offices à l'église se faisaient aussi en soureth dans leur majorité, avec quelques ajouts mineurs en arabe. On remarque toujours d'ailleurs chez les 
femmes, même jeunes, qui n'ont pas ou peu suivi un cursus scolaire des problèmes de locution en arabe.

Toute fille, dès le plus jeune âge, devait aider les femmes adultes dans les tâches ménagères quotidiennes, s'occuper de ses frères et ne sortait de la maison qu'en leur compagnie ou en compagnie de la mère. Les lieux de sorties étaient limités aux réunions familiales exclusivement féminines, au marché et aux différentes activités et offices à l'église du village, cela toujours en compagnie de frères ou d'une femme adulte. L'éducation des filles se passait dans les écoles publiques du village réservées aux filles et ne dépassait guère le cycle primaire ou complémentaire, comme partout en Irak ${ }^{23}$, considéré par les parents comme suffisant pour les filles destinées au mariage, sauf quelques rares cas qui poursuivaient des études secondaires et universitaires. Les vendredis, jours fériés en Irak, une éducation parallèle pour les enfants et les jeunes adolescents se faisait dans les locaux de l'église du village, sous forme de cours de catéchisme, mais incluait des cours de cuisine et de couture pour les filles. Les classes à l'église étaient divisées par tranches d'âges et supervisées par les prêtres et les religieuses pour éviter toute mixité entre les sexes. Les offices religieux suivaient aussi cette ségrégation par sexe dans l'église, sauf pour la chorale qui était le seul lieu de mixité contrôlée.

Le mariage s'effectuait de façon traditionnelle et endogamique entre l'âge de 14 et 16 ans avec un conjoint choisi par le père, dans l'entourage direct de la famille (parents, voisins). À travers l'union matrimoniale se forgeait des alliances entre les différentes familles et se nouait la solidarité sociale du Clan. Comme chez leurs voisins musulmans, l'époux devait présenter un mahr (douaire) ${ }^{24}$ en biens, argent, joaillerie, animaux, etc. aux parents en compensation de leur fille. Suite au mariage, la jeune épouse habitait en général la même maison 
avec les beaux-parents ou dans une maison mitoyenne. La nouvelle mariée se trouvait ainsi limitée à la procréation et aux tâches ménagères quotidiennes. Dans certains cas, suite au décès du mari, on a pu observer à travers les documents officiels un remariage avec un frère du défunt.

Le divorce et la répudiation étaient presque inexistants dans les villages chrétiens, où toute femme divorcée ou répudiée se trouvait obligée de rentrer chez ses parents et devenait marginalisée socialement par la communauté. Les rares cas de femmes qui s'émancipaient de cette tutelle familiale se trouvaient marginalisées et répudiées socialement par la communauté, au point de devoir quitter le village et s'installer en ville. Cette marginalisation touchait aussi les femmes qui poursuivaient des études ou qui avaient un emploi.

Suite à la fuite des familles en 2014 face à l'invasion des villages par le groupe «État islamique » (Daesh), l'installation des réfugiés dans les camps de caravanes autour d'Arbil et de Dohuk en Irak s'est faite sur la base de l'appartenance villageoise et familiale. Ainsi, chaque camp était réservé aux habitants d'un même village où la même famille patriarcale partageait les caravanes mitoyennes. Ce n'est qu'avec l'exil vers les pays limitrophes (Turquie, Jordanie, Liban) et l'habitat en zone urbaine que la famille patriarcale s'est trouvée disloquée et éparpillée.

\section{Les processus d'émancipation des chrétiennes d'Irak réfugiées au Liban}

Les premières familles chrétiennes irakiennes des villages de la plaine de Ninive sont venues au Liban en 2014. Vivant dans un contexte de précarité économique nouvelle, elles ont essayé au début de s'agglomérer dans un même quartier, dans les régions de Sad el Bouchrieh et Dekweneh et leurs environs dans la banlieue nord-est de Beyrouth notamment, pour conserver l'emprise de la famille patriarcale. Cependant, il est impossible d'habiter en groupe compact en zone urbaine, où les immeubles et les appartements sont inadéquats. De plus, suite à la hausse de la demande due à l'arrivée massive des réfugiés irakiens et syriens, les tarifs de location ${ }^{25}$ sont devenus exorbitants. La majorité des familles patriarcales nombreuses ont été contraintes de se délocaliser et de s'éparpiller vers d'autres quartiers limitrophes ou lointains, au gré des possibilités de location. D’autres familles nucléaires ont aussi choisi de s'installer au Liban, loin de leur famille patriarcale en majorité restée en Irak. En dernier lieu, le processus d'inscription comme réfugiés au HCR oblige aussi les familles à s'enregistrer par familles nucléaires en vue d'une possible réinstallation dans un pays tiers. Ce processus a permis la dislocation de la famille patriarcale en multiples groupes avec chacun une destination et une date de départ différente.

Cet éloignement géographique modifie les rôles de la femme dans la famille nucléaire et lui permet de commencer à s'émanciper de la tutelle de la famille patriarcale. Ceci reste surtout vrai dans les familles nucléaires dont la famille patriarcale est restée au pays ou a été disloquée par le processus de réinstallation du HCR. De même, la précarité et l'appauvrissement progressif des ménages ont pousséles femmes à rechercher du travail pour subvenir aux besoins du foyer, car les hommes en situation illégale étaient dans l'impossibilité de trouver du travail.

Or, dans les pays musulmans du Moyen-Orient, dont l'Irak, les femmes ont toujours une faible participation au marché du travail ${ }^{26}$. Le travail a beaucoup aidé à leur émancipation à l'égard de la famille patriarcale, car il était plus facile de trouver des emplois pour les jeunes femmes qu'à leurs parents âgés ou leurs conjoints. Ceci est principalement dû à la demande sur le marché local du travail, mais aussi à la facilité qu'ont les femmes réfugiées à circuler, contrairement aux hommes qui ont peur des contrôles de police à cause de leur situation illégale.

Comme dans le cas des femmes turques issues du milieu rural vivant en Europe, « le fait de gagner leur 
vie bouleverse les normes traditionnelles selon lesquelles la femme, à la campagne, était considérée surtout, comme une aide à disposition du mari ou de la famille. En tant qu'immigrées, les femmes prennent conscience de leur capacité à travailler et à vivre de façon autonome ${ }^{27}$ ». Au Liban ce schéma est le même, comme le constate l'étude conduite auprès des réfugiés chrétiens irakiens de Beyrouth. Ainsi, plusieurs femmes, auparavant limitées au foyer et sans éducation avancée, ont commencé à travailler dans des magasins, des ateliers de confection et des usines dans les régions commerciales et industrielles de la banlieue nord-est de Beyrouth, tandis que les hommes au chômage (surtout ceux âgés de 40 et plus) passent leur temps dans les cafés. Parmi ces femmes présentes sur le marché du travail, on trouve aussi beaucoup de filles mineures, âgées entre 13 et 18 ans, n'ayant pas la possibilité de suivre le cursus scolaire libanais, vu le problème de langue et de culture. Cette «mutation du rôle et des responsabilités de chacun au sein de la cellule familiale consacre les femmes et les enfants comme piliers de la sécurité économique des ménages ${ }^{28}$ ".

Au Liban, les règles du mariage chez les réfugiés chrétiens irakiens de la plaine de Ninive ont subi un changement radical : les églises locales imposent l'âge de 18 ans pour marier les filles et le douaire n'y est pas permis. Cependant, le mariage traditionnel a pu s'adapter aux lois libanaises concernant l'âge tout en gardant des traits irakiens, notamment dans la forme des festivités qui l'accompagnent. Depuis 2014, on a pu remarquer une augmentation des cas de mariage sans l'aval des parents avec des Irakiens d'autres régions ou avec des Libanais, car la contraction d'unions avec des personnes étrangères à leur communauté d'origine, surtout si les conjoints sont des autochtones, leur permet "d'obtenir ainsi un statut stable ${ }^{29}$ ". Toutefois, ces mariages « mixtes » restent l'exception au sein de la communauté des réfugiés chrétiens irakiens de la plaine de Ninive.
De même, on a pu aussi remarquer une dislocation des familles traditionnelles, à cause d'un divorce ou de la répudiation survenus dans les pays d'exil. Ce constat est appuyé par les registres des tribunaux ecclésiastiques chaldéens, syriaques et assyriens au Liban et en Turquie. Ce phénomène se remarque surtout chez les familles nucléaires, exilées loin de la famille patriarcale, suite à la paupérisation et à lémancipation de la femme par le travail, devenue la source essentielle du revenu familial. Des familles où il y a donc eu un inversement des rôles entre l'homme et la femme dans la gestion du ménage.

\section{Vers une inversion des rôles genrés}

Ce changement dans les coutumes et les mœurs, ainsi que l'émancipation des filles par rapport à la famille, reste mal vécu par les parents et les maris habitués à des rituels traditionnels spécifiques dans leur village. Ainsi, «les hommes doivent composer avec la nouvelle visibilité de leurs épouses ou de leurs filles qui, en immigration, commencent à sortir du cadre familial et de voisinage $e^{30} »$.

Selon le Bureau de la coordination des affaires humanitaires des Nations unies, L'étude de 2016 montre ainsi qu'une femme irakienne gagne $400 \$$ par mois en moyenne dans le travail dans les magasins, les ateliers de confection et les usines. L'homme, s'il travaille, ne gagne en moyenne que 300 \$ par mois, quel que soit le travail qu'il effectue. pas moins de $80 \%$ des déplacés internes et réfugiés à travers le monde sont des femmes et des enfants. Ce chiffre est presque le même chez les familles chrétiennes irakiennes d'origine rurale installées au Liban, où les femmes et les enfants forment les deux tiers du nombre total des réfugiés irakiens chrétiens qui, selon les chiffres disponibles, atteindrait 25000 personnes.

Concernant les réfugiés chrétiens irakiens de Beyrouth, létude citée ci-dessus a montré que 80 \% 
des 500 ménages visités comptait sur le revenu du travail des femmes pour subsister. Mais l'étude montre aussi un nombre croissant de familles monoparentales ${ }^{31}, 58$ ménages $^{32}$ au total, où la mère est devenue le chef de ménage. Les causes sont multiples : le père est absent, décédé ou resté (ou reparti) en Irak, ou il y a eu un divorce entre 2014 et 2016. Ainsi, selon Isabelle Rigoni, «les valeurs traditionnelles sont perturbées par la concurrence de nouvelles normes et l'on assiste à une inversion des rôles. La femme gagne l'argent du ménage et devient, financièrement, le chef de famille ${ }^{33}$ ».

Le nombre de femmes chrétiennes irakiennes sur le marché du travail au Liban est actuellement deux fois supérieur au nombre d'hommes, souvent sans travail ou ayant un travail sous-payé. Létude de 2016 montre ainsi qu'une femme irakienne gagne 400 \$ par mois en moyenne dans le travail dans les magasins, les ateliers de confection et les usines. L'homme, s'il travaille, ne gagne en moyenne que 300 \$ par mois, quel que soit le travail qu'il effectue.

\section{Conclusion}

Comme le montrent plusieurs études concernant les mutations des rôles et l'inversement des rôles entre l'homme et la femme dans la gestion du ménage suite à l'immigration, les rôles des femmes d'origine rurale réfugiées en milieu urbain ont beaucoup évolué, à l'instar des chrétiennes irakiennes arrivées au Liban après 2014. Dans le pays d'origine, suivant Mirjana Morokvasic, «le modèle en vigueur del'homme pourvoyeur de ressources et de la femme au foyer ne permettait pas de voir la femme autrement que dans un statut de dépendance, à charge ${ }^{34}$ ». Cependant, de simple femme au foyer sans aucune autorité dans la direction du ménage, la femme dans le pays d'exil ou d'accueil, par son apport financier, tend à en prendre la tête et même y remplacer l'homme, souvent absent ou sans travail.

L'émancipation de la tutelle du mari et de la famille patriarcale a surtout été accélérée par le biais de la participation financière de la femme au revenu de la famille. Cette participation lui a donné un atout qui lui manquait dans le pays d'origine, où elle subissait la tutelle de l'homme seul pourvoyeur financier du ménage. Ainsi, on a pu noter "qu'en matière de santé et d'éducation les femmes chefs de ménage investissent davantage dans leurs enfants en termes de ressources, de temps et de support émotionnel que ne le font les hommes ${ }^{35}$ ".

Depuis les années 1990, de «multiples mécanismes d'invisibilisation contribuent à occulter une bonne partie de l'existence des migrantes et des immigrées, notamment la pluralité de leur activités économiques ${ }^{36}$ ". Il reste cependant le fardeau social que la femme émancipée de la tutelle de son entourage masculin doit supporter pour survivre aux années d'exil, à la réinstallation dans un nouveau pays ou aux aléas d'un retour possible vers le pays d'origine. 